\title{
APPROXIMATE ENTROPY AND MUTUAL INFORMATION ANALYSIS OF THE ELECTROENCEPHALOGRAM IN ALZHEIMER'S DISEASE PATIENTS
}

\author{
D. Abásolo ${ }^{1}$, R. Hornero ${ }^{1}$, J. Escudero ${ }^{1}$, C. Gómez ${ }^{1}$, M. García ${ }^{1}$ and M. López ${ }^{1}$ \\ ${ }^{1}$ E.T.S. Ingenieros de Telecomunicación, University of Valladolid, Valladolid, Spain \\ danaba@tel.uva.es
}

\begin{abstract}
The aim of this study was to analyse the EEG background activity in Alzheimer's disease (AD) with two non-linear methods: Approximate Entropy (ApEn) and Auto Mutual Information $(A M I)$. ApEn quantifies the regularity in data, while $A M I$ detects linear and nonlinear dependencies in time series and can be used as a normalized complexity measure. EEGs were recorded from the 19 scalp loci of the international 10-20 system in $11 \mathrm{AD}$ patients and 11 age-matched controls. ApEn was significantly lower in AD patients at electrodes O1, P3 and P4 $(p<0.05)$. The $A M I$ of the AD patients decreased significantly more slowly with time delays than the AMI of control subjects at electrodes T5, O1, $\mathrm{P} 3$ and $\mathrm{P} 4(p<0.05)$. The decreased irregularity and complexity found in $\mathrm{AD}$ patients leads us to think that EEG analysis with $A p E n$ and $A M I$ could help to increase our insight into brain dysfunction in AD.
\end{abstract}

Keywords: Alzheimer's disease, electroencephalogram, approximate entropy, mutual information.

\section{INTRODUCTION}

Alzheimer's disease (AD) is considered to be the main cause of dementia in western countries [1]. $\mathrm{AD}$ is characterized by progressive impairments in cognition and memory whose course lasts several years prior to death [2]. These clinical features are accompanied by histological changes in the brain, which include widespread cortical atrophy, intracellular deposition of neurofibrillary tangles and extracellular deposition of senile plaques, particularly in the hippocampus and the cerebral cortex [2]. Although a definite diagnosis is only possible by necropsy, a differential diagnosis with other types of dementia and with major depression should be attempted. Magnetic resonance imaging and computerized tomography can be normal in the early stages of $\mathrm{AD}$ but a diffuse cortical atrophy is the main sign in brain scans. Mental status tests are also useful.

The electroencephalogram (EEG) has been used as a tool for diagnosing dementias for several decades. The hallmark of EEG abnormalities in AD patients is a shift of the power spectrum to lower frequencies and a decrease of coherence among cortical areas [2], although in the early stages of the disease the EEG may exhibit normal frequencies [3]. Recent progress in the theory of non-linear dynamics has provided new methods for the study of the EEG [2]. Non-linearity is present in many dynamical systems found in nature, including the brain. Given the highly non-linear nature of the neuronal interactions at multiple levels of spatial scales, the EEG appears to be an appropriate area for non-linear time series analysis [2].

Several studies have examined the non-linear dynamics of the EEG in AD. It has been found that $\mathrm{AD}$ patients have lower correlation dimension $\left(D_{2}\right)$ values a measure of dimensional complexity of the underlying system - than control subjects [4-6]. Moreover, the first Lyapunov exponent ( $L 1)$ has also been used to characterize non-linear behaviour in the brain and it has been shown that $\mathrm{AD}$ patients have significantly lower $L 1$ values than controls in almost all EEG channels [4, 5]. However, estimating the non-linear dynamical complexity of physiological data using measures such as $D_{2}$ and $L 1$ is problematic, as the amount of data required for meaningful results in their computation is beyond the experimental possibilities for physiological data [7]. Thus, it becomes necessary to study the EEG background activity with more suitable methods.

The present study was undertaken to examine the EEG background activity in AD with two different nonlinear methods: Approximate Entropy (ApEn) and Auto Mutual Information $(A M I)$. While $A p E n$ is a family of statistics that quantifies the regularity in data [8], AMI detects linear and non-linear dependencies in a time series [9]. We wanted to test the hypothesis that the non-linear characteristics of the EEG background activity in AD patients' EEG would be different than those of age-matched controls, hence indicating an abnormal type of dynamics.

\section{MATERIALS AND METHODS}

\section{Subjects and signals}

Twenty-two subjects participated in this study. Eleven patients ( 5 men and 6 women; age $=72.5 \pm 8.3$ years, mean \pm standard deviation SD) fulfilling the criteria of probable $\mathrm{AD}$ were recruited from the Alzheimer's Patients' Relatives Association of Valladolid (AFAVA) and referred to the University Hospital of Valladolid (Spain), where the EEG was recorded. All of them had undergone a thorough clinical evaluation that included clinical history, physical and neurological examinations, brain scans and a Mini-Mental State Examination (MMSE), generally accepted as a quick and simple way to evaluate cognitive function [10]. The mean MMSE score for the patients was $13.1 \pm 5.9$ (Mean \pm SD). Five of them had a score of less than 12 points, indicating a severe degree of dementia.

The control group consisted of 11 age-matched control subjects without past or present neurological 
disorders ( 7 men and 4 women; age $=72.8 \pm 6.1$ years, mean \pm SD). The MMSE score value was 30 for all control subjects. The local ethics committee approved the study. All control subjects and all caregivers of the patients gave their informed consent for participation in the current study.

\section{EEG recording}

More than five minutes of EEG data from each subject were recorded with a Profile Study Room 2.3.411 EEG equipment (Oxford Instruments) at electrodes F3, F4, F7, F8, Fp1, Fp2, T3, T4, T5, T6, C3, C4, P3, P4, O1, $\mathrm{O} 2, \mathrm{Fz}, \mathrm{Cz}$ and $\mathrm{Pz}$ of the international 10-20 system. The sample frequency was $256 \mathrm{~Hz}$, with a 12-bit A-to-D precision. Recordings were made with the subjects in a relaxed state and under the eyes-closed condition. All EEGs were visually inspected by a specialist physician to check for eye movement and other artefacts. Afterwards, EEGs were organized in 5 second artefactfree epochs (1280 points) that were copied as ASCII files for off-line analysis on a personal computer. Furthermore, all recordings were digitally filtered with a band-pass filter with cut-off frequencies at $0.5 \mathrm{~Hz}$ and at $40 \mathrm{~Hz}$ in order to remove electromyographic activity prior to the ApEn and $A M I$ calculations.

\section{Approximate entropy (ApEn)}

Approximate Entropy (ApEn) is a family of statistics introduced to provide a widely applicable, statistically valid formula that will distinguish data sets by a measure of regularity [11]. It is scale invariant and model independent and it discriminates series for which clear feature recognition is difficult [8]. Moreover, $A p E n$ can be applied to short time series and it is finite for stochastic, noisy deterministic and composite processes [8].

ApEn assigns a non-negative number to a time series, with larger values corresponding to more irregularity in the data. Two input parameters, a run length $m$ and a tolerance window $r$, must be specified. Briefly, ApEn measures the logarithmic likelihood that runs of patterns that are close (within $r$ ) for $m$ contiguous observations remain close (within the same tolerance width $r$ ) on subsequent incremental comparisons. $\operatorname{ApEn}(m, r, N)$, where $N$ is the number of points of the time series, must be considered a family of characterizing measures: comparisons between time series can only be made with the same values of $m, r$ and $N[8]$.

Formally, given $N$ data points from a time series $\{x(n)\}=x(1), x(2), \ldots, x(N)$, one should follow the following steps to compute ApEn [8]:
1. Form $N-m+1$ vectors $X(1) \ldots X(N-m+1)$ defined by: $X(i)=[x(i), x(i+1), \ldots, x(i+m-1)], i=1 \ldots N-m+1$.

2. Define the distance between $X(i)$ and $X(j)$, $d[X(i), X(j)]$, as the maximum absolute difference between their respective scalar components, i.e. the maximum norm:

$$
d[X(i), X(j)]=\max _{k=1,2, \ldots, m}|x(i+k-1)-x(j+k-1)| .
$$

3. For a given $X(i)$, count the number of $j(j=1 \ldots N$ $m+1, j \neq i)$ so that $d[X(i), X(j)] \leq r$, denoted as $N^{m}(i)$. Then, for $i=1 \ldots N-m+1$,

$$
C_{r}^{m}(i)=N^{m}(i) /(N-m+1) .
$$

4. Compute the natural logarithm of each $C_{r}^{m}(i)$, and average it over $i$,

$$
\phi^{m}(r)=\frac{1}{N-m+1} \sum_{i=1}^{N-m+1} \ln C_{r}^{m}(i) .
$$

5. Increase the dimension to $m+1$. Repeat steps 1) to 4) and find $C_{r}^{m+1}(i)$ and $\phi^{m+1}(r)$.

6. We define ApEn by:

$$
\operatorname{ApEn}(m, r, N)=\phi^{m}(r)-\phi^{m+1}(r) .
$$

Preliminary evidence suggests that applied to EEGs ApEn may be predictive of epileptic seizures [12] and may be an effective tool to quantify the depth of anaesthesia [13].

Although $m$ and $r$ are critical in determining the outcome of $A p E n$, no guidelines exist for optimising their values. In this pilot study, ApEn was estimated with $m=1$ and $r=0.25$ times the SD of the original data sequence. Normalizing $r$ in this manner gives ApEn a translation and scale invariance, in that it remains unchanged under uniform process magnification, reduction, or constant shift to higher or lower values [8]. It has been demonstrated [8] that these input parameters produce good statistical reproducibility for $A p E n$ for time series of length $N \geq 60$, as considered herein.

\section{Auto Mutual Information (AMI)}

Mutual information $(M I)$ provides a measure of both the linear and non-linear statistical dependencies between two time series [9]. The $M I$ between measurement $x_{i}$ and measure $y_{i}$ is the amount of information that the former provides about the latter. The $M I$ between two measurements taken from a single time series $x(t)$ separated by time $\tau$ is called the auto mutual information $(A M I)$. The $A M I$ estimates, on average, the degree to which $x(t+\tau)$ can be predicted from $x(t)$.

The $A M I$ between $x(t)$ and $x(t+\tau)$ is [9]:

$$
I_{X X_{\tau}}=\sum_{x(t), x(t+\tau)} P_{X_{\tau}}[x(t), x(t+\tau)] \cdot \ln \left\{\frac{P_{X X_{\tau}}[x(t), x(t+\tau)]}{P_{X}[x(t)] \cdot P_{X_{\tau}}[x(t+\tau)]}\right\} .
$$


$P_{X}[x(t)]$ is the normalized histogram of the distribution of values observed for $x(t)$, while $P_{X X_{\tau}}[x(t), x(t+\tau)]$ is the joint probability density for the measurements of $x(t)$ and $x(t+\tau)$. The rate of decrease of the AMI with increasing $\tau$ is a normalized complexity measure of the time series [9].

The $M I$ of the EEG has been used to describe the information transmission in the brain in $\mathrm{AD}$ [9] and in schizophrenia [14]. Moreover, it has been shown that it might be useful to predict the response to anaesthesia [15].

In this pilot study we obtained the AMI of the EEG from both $\mathrm{AD}$ patients and control subjects for time delays between 0 and $0.5 \mathrm{~s}$. The rate of decrease of the $A M I$, estimated using a least-squares fitting method and computed from a time delay 0 to the first minimum value, was used as a measure of complexity.

\section{RESULTS}

ApEn was estimated for channels F3, F4, F7, F8, Fp1, Fp2, T3, T4, T5, T6, C3, C4, P3, P4, O1 and O2 with $m=1$ and $r=0.25$ times the $\mathrm{SD}$ of the original data sequence. The results are summarized in table 1 . Statistical differences between groups at the different electrodes were evaluated with Student's $t$-test. A Bonferroni correction was used in order to account for multiple comparisons. It can be seen that $\mathrm{AD}$ patients had lower $A p E n$ values at 15 electrodes, with significant differences between both groups $(p<0.05)$ at electrodes P3, P4 and O1. These results suggest that EEG activity of $\mathrm{AD}$ patients is more regular in the parietal and left occipital regions than in a normal brain.

Fig. 1 represents the normalized average $A M I$ curves of the control subjects and $\mathrm{AD}$ patients at electrode $\mathrm{O} 2$. Both profiles decrease gradually with increasing values of the time delay $\tau$. The mean rates of decrease of the $A M I$ curves for all electrodes are summarized in table 2. The statistical differences were tested in the same way as with ApEn (Student's $t$-test and Bonferroni correction for multiple comparisons). These results show that, with the exception of electrode T4, the AMI decreases more

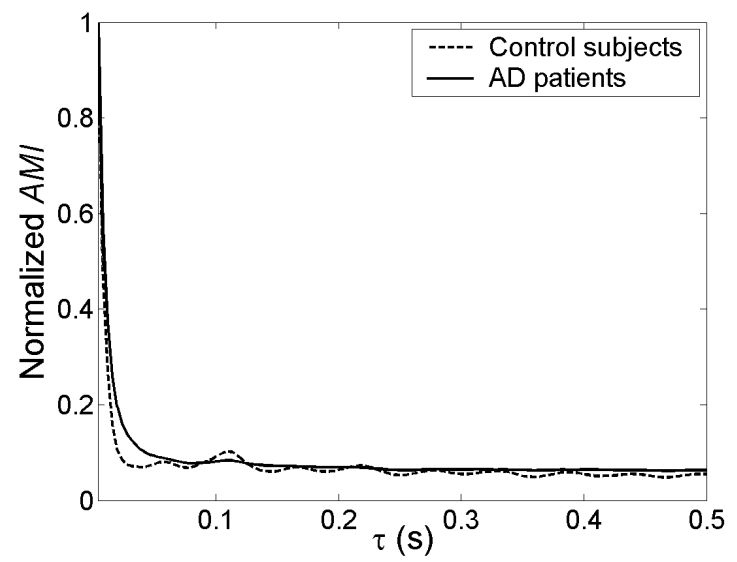

Fig. 1 Normalized $A M I$ curves of the 11 control subjects and $11 \mathrm{AD}$ patients at electrode $\mathrm{O} 2$.

\begin{tabular}{c|cc} 
Electrode & $\begin{array}{c}\text { Control subjects } \\
(\text { Mean } \pm \text { SD })\end{array}$ & $\begin{array}{c}\text { AD patients } \\
(\text { Mean } \pm \text { SD })\end{array}$ \\
\hline F3 & $0.7378 \pm 0.1821$ & $0.6288 \pm 0.1181$ \\
F4 & $0.7100 \pm 0.2028$ & $0.6933 \pm 0.1371$ \\
F7 & $0.7732 \pm 0.2072$ & $0.7349 \pm 0.1634$ \\
F8 & $0.7867 \pm 0.1775$ & $0.7309 \pm 0.1563$ \\
Fp1 & $0.7182 \pm 0.1649$ & $0.5641 \pm 0.2006$ \\
Fp2 & $0.6994 \pm 0.2194$ & $0.5745 \pm 0.1363$ \\
T3 & $0.9580 \pm 0.2869$ & $0.9236 \pm 0.2472$ \\
T4 & $0.9296 \pm 0.2485$ & $0.9342 \pm 0.3186$ \\
T5 & $0.9125 \pm 0.1953$ & $0.6936 \pm 0.2081$ \\
T6 & $0.8976 \pm 0.2018$ & $0.6914 \pm 0.2179$ \\
C3 & $0.8363 \pm 0.1670$ & $0.7291 \pm 0.1954$ \\
C4 & $0.8490 \pm 0.1384$ & $0.7703 \pm 0.2150$ \\
P3* & $0.8599 \pm 0.1331$ & $0.6088 \pm 0.1817$ \\
P4* & $0.8644 \pm 0.1320$ & $0.6423 \pm 0.1753$ \\
O1* & $0.9714 \pm 0.1801$ & $0.6989 \pm 0.1939$ \\
O2 & $0.9357 \pm 0.2051$ & $0.6867 \pm 0.1961$
\end{tabular}

Table 1: average $\operatorname{ApEn}(m=1, r=0.25)$ values of the EEGs for both groups of subjects in all channels. Significant differences $(p<0.05)$ are marked with an asterisk.

\begin{tabular}{c|cc} 
Electrode & $\begin{array}{c}\text { Control subjects } \\
\text { (Mean } \pm \text { SD) }\end{array}$ & $\begin{array}{c}\text { AD patients } \\
(\text { Mean } \pm \text { SD })\end{array}$ \\
\hline F3 & $-12.46 \pm 3.83$ & $-9.88 \pm 1.73$ \\
F4 & $-12.72 \pm 2.90$ & $-10.69 \pm 2.43$ \\
F7 & $-12.64 \pm 3.81$ & $-11.62 \pm 3.53$ \\
F8 & $-13.33 \pm 3.53$ & $-11.70 \pm 3.45$ \\
Fp1 & $-12.54 \pm 3.34$ & $-9.09 \pm 2.34$ \\
Fp2 & $-11.87 \pm 3.72$ & $-8.49 \pm 2.20$ \\
T3 & $-15.15 \pm 4.64$ & $-14.62 \pm 5.12$ \\
T4 & $-14.97 \pm 3.94$ & $-15.19 \pm 6.60$ \\
T5* & $-14.83 \pm 3.51$ & $-9.68 \pm 2.92$ \\
T6 & $-14.43 \pm 3.33$ & $-10.39 \pm 3.33$ \\
C3 & $-13.91 \pm 3.02$ & $-11.98 \pm 3.17$ \\
C4 & $-14.25 \pm 3.26$ & $-12.63 \pm 3.63$ \\
P3* & $-14.48 \pm 2.84$ & $-9.43 \pm 2.70$ \\
P4* & $-14.66 \pm 2.72$ & $-9.98 \pm 2.64$ \\
O1* & $-15.61 \pm 3.45$ & $-10.28 \pm 3.25$ \\
O2 & $-14.57 \pm 3.65$ & $-10.15 \pm 3.24$
\end{tabular}

Table 2: average values of the rate of decrease of the $A M I$ for both study groups in all channels. Significant differences $(p<0.05)$ are marked with an asterisk.

slowly in $\mathrm{AD}$ patients, with significant differences $(p<0.05)$ at $\mathrm{T} 5, \mathrm{P} 3, \mathrm{P} 4$ and $\mathrm{O} 1$.

\section{DISCUSSION AND CONCLUSIONS}

In this pilot study we have applied the ApEn and the $A M I$ to analyse the EEG background activity in AD. $A p E n$ is a family of statistics that quantifies the regularity in time series, with increasing values corresponding to more irregularity [8]. On the other hand, $A M I$ estimates the degree to which $x(t+\tau)$ can be 
predicted from $x(t)$. Moreover, the rate of decrease of the $A M I$ with increasing $\tau$ is a normalized complexity measure of the time series [9]. Furthermore, ApEn and $A M I$ do not require a large number of data points to be reliably estimated and can be applied to non-stationary time series [8,9]. Thus, this set of measures is much better suited for EEG analysis than traditional nonlinear techniques as $L 1$ or $D_{2}$.

We have found that the ApEn of the EEG was lower in AD patients than in control subjects, with significant differences at electrodes P3, P4, and O1 $(p<0.05)$. Furthermore, we have also found that the $A M I$ decreases more slowly with $\tau$ in AD patients, with significant differences in the slopes at T5, P3, P4 and O1 $(p<0.05)$. Thus, we can infer that brains affected by AD show a more regular and less complex electrophysiological behaviour. These results are consistent with previous studies showing that the EEG of AD patients is less complex [4-6, 9] and more regular [16] than in agematched control subjects. The increased regularity and reduced complexity in the AD patients' EEG could be explained by a decrease of dynamical complexity in the brain. However, the implications of this decreased EEG irregularity or complexity are not clear. Among others, three mechanisms can be responsible for it: neuronal death, a general effect of neurotransmitter deficiency and loss of connectivity of local neural networks [2].

Although our results indicate that ApEn and AMI could be useful tools to help in the diagnosis of $\mathrm{AD}$, further studies with a larger sample size must be carried out to prove the clinical applications of both methods. Future lines of research include the study of the correlations between $A p E n$ and $A M I$ results and the characterization of the EEG background activity in other dementias to check if the reported findings are specific to $\mathrm{AD}$.

\section{ACKNOWLEDGMENTS}

This study was partially supported by a project from the "Consejería de Fomento de la Junta de Castilla y León" and "Ministerio de Educación y Ciencia" and FEDER grant MTM 2005-08519-C02-01.

\section{REFERENCES}

[1] Bird T.D. (2001): 'Alzheimer's disease and other primary dementias' in Harrison's principles of internal medicine, Braunwald E. et al (Eds), The McGraw-Hill Companies Inc, New York, NY, pp. 2391-2399.

[2] Jeong J. (2004): 'EEG dynamics in patients with Alzheimer's disease', Clinical Neurophysiology, 115, pp. 1490-1505.

[3] Markand O.N. (1990): 'Organic brain syndromes and dementias' in Current Practice of Clinical Electroencephalography, Daly D.D. and Pedley T.A. (Eds), Raven Press, New York, NY, pp. 401423 .
[4] Jeong J., Kim S.Y. and Han S.H. (1998): 'Nonlinear dynamical analysis of the EEG in Alzheimer's disease with optimal embedding dimension', Electroencephalography and Clinical Neurophysiology, 106, pp. 220-228.

[5] Jeong J., Chae J.H., Kim S.Y. and Han S.H. (2001): 'Nonlinear dynamic analysis of the EEG in patients with Alzheimer's disease and vascular dementia', Journal of Clinical Neurophysiology, 18, pp. 58-67.

[6] Stam C.J., Jelles B., Achtereekte H.A.M., Rombouts S.A.R.B., Slaets J.P.J. and Keunen R.W.M. (1995): 'Investigation of EEG nonlinearity in dementia and Parkinson's disease', Electroencephalography and Clinical Neurophysiology, 95, pp. 309-317.

[7] Eckmann J.P. and Ruelle D. (1992): Fundamental limitations for estimating dimensions and Lyapunov exponents in dynamical systems', Physica D, 56, pp. 185-187.

[8] Pincus S.M. (2001): 'Assessing serial irregularity and its implications for health', Annals of the New York Academy of Sciences, 954, pp. 245-267.

[9] Jeong J., Gore J.C. and Peterson B.S. (2001): 'Mutual information analysis of the EEG in patients with Alzheimer's disease', Clinical Neurophysiology, 112, pp. 827-835.

[10] Folstein M.F., Folstein S.E. and McHugh P.R. (1975): 'Mini-mental state. A practical method for grading the cognitive state of patients for the clinician', Journal of Psychiatric Research, 12, pp. 189-198.

[11] Pincus S.M. (1991): 'Approximate entropy as a measure of system complexity', Proceedings of the National Academy of Sciences of the USA, 88, pp. 2297-2301.

[12] Radhakrishnan N. and Gangadhar B.N. (1998): 'Estimating regularity in epileptic seizure timeseries data. A complexity-measure approach', IEEE Engineering in Medicine and Biology Magazine, 17, pp. 89-94.

[13] Zhang X.-S. and Roy R.J. (2001): 'Derived fuzzy knowledge model for estimating the depth of anesthesia', IEEE Transactions on Biomedical Engineering, 48, pp. 312-323.

[14] Na S.H., Jin S.-H., Kim S.Y. and Ham B.-J. (2002): 'EEG in schizophrenic patients: mutual information analysis', Clinical Neurophysiology, 113, pp. 1954-1960.

[15] Huang L., Yu P., Ju F. and Cheng J. (2003): 'Prediction of response to incision using the mutual information of electroencephalogram during anaesthesia', Medical Engineering and Physics, 25, pp. 321-327.

[16] Abásolo D., Hornero R., Espino P., Poza J., Sánchez C.I. and de la Rosa R. (2005): 'Analysis of regularity in the EEG background activity of Alzheimer's disease patients with Approximate Entropy', Clinical Neurophysiology, 116, pp. 1826-1834. 